\title{
Formatividad y producción en las estéticas de Luigi Pareyson y Gianni Vattimo*
}

\author{
Training and production in the aesthetics of \\ Luigi Pareyson and Gianni Vattimo
}

Formatividade e produção nas estéticas de Luigi Pareyson e Gianni Vattimo

Luis Uribe Miranda**

\begin{abstract}
RESUMEN
Los conceptos de "formatividad" y "producción" caracterizan las

estéticas de Luigi Pareyson y Gianni Vattimo, respectivamente, y forman parte del proceso de renovación de la estética italiana a partir de la segunda mitad del siglo pasado. El énfasis en la formatividad y la producción permite a los autores afirmar el carácter filosófico de la estética, cuya teoría se nutre de la materia prima proporcionada por las experiencias artísticas concretas. A partir del análisis de los textos originales, primero de Pareyson y después de Vattimo, se muestra el carácter ontológico de la estética. El texto de Gianni Vattimo, de 1961, Il concetto di fare in Aristotele, es la base para afirmar la tesis según la cual la estética posee una vocación ontológica, anticipando lo expresado por el mismo Vattimo en Ontologia e poesia, de 1967, aspecto que hace patente la comparación con Luigi Pareyson.
\end{abstract}

Palabras clave: estética, formatividad, producción, Luigi Pareyson, Gianni Vattimo.

\footnotetext{
* Este artículo es fruto del Proyecto de Investigación PVCHU332-2016: Estética e hermenêutica na filosofia italiana contemporânea: um diálogo entre Luigi Pareyson e Gianni Vattimo coordinado por el autor y desarrollado con el patrocinio de la Universidade Federal do Maranhão (UFMA).

** Chileno. Doctor en Filosofía por la Università degli Studi di Torino, Italia. Posdoctorado en Filosofía Política por la Università degli Studi di Torino, Italia (2019) y en Estética, por la Universidade Federal de Minas Gerais (UFMG), Brasil (2015). Profesor del Departamento de Filosofía de la Universidade Federal do Maranhão (UFMA), Brasil. Coordinador del Grupo de Estudios e Investigación en Filosofia Italiana (GEPFIT), registrado en CNPq, Profesor permanente del Magíster en Filosofía y Co-Director de la Coleção Filosofia Italiana de la Editorial Paco de São Paulo, Brasil. Contacto: luis.uribe@ufma.br ORCID: http://orcid.org/0000-0002-0765-7932
} 


\section{SUMMARY}

The concepts of "training" and "production" characterize the aesthetics of Luigi Pareyson and Gianni Vattimo, respectively, and are part of the process of renewal of Italian aesthetics from the second half of the last century. The emphasis on training and production allows the authors to affirm the philosophical character of aesthetics, whose theory is nourished by the raw material provided by concrete artistic experiences. From the original texts' analysis, first by Pareyson and then by Vattimo, the ontological character of aesthetics is shown. Gianni Vattimo's 1961 text, Il concetto di fare in Aristotele, is the basis for affirming the thesis according to which aesthetics has an ontological vocation, anticipating what Vattimo himself expressed in Ontologia e Poesia, 1967, an aspect that makes the comparison with Luigi Pareyson clear.

\section{RESUMO}

Os conceitos de "formatividade" e "produção" caracterizam as estéticas de Luigi Pareyson e Gianni Vattimo, respectivamente, e fazem parte do processo de renovação da estética italiana a partir da segunda metade do século passado. A ênfase na formatividade e na produção permite aos autores afirmarem o caráter filosófico da estética, cuja teoria é alimentada pela matéria-prima proporcionada pelas experiências artísticas concretas. A partir da análise dos textos originais, primeiro de Pareyson e depois de Vattimo, apresenta-se o caráter ontológico da estética. O texto de Gianni Vattimo, de 1961, Il concetto di fare in Aristotele, é a base para fundamentar a teoria de que a estética possui uma vocação ontológica, antecipando o que o próprio Vattimo expressou em Ontologia e poesia, de 1967, um aspecto que torna clara a comparação com Luigi Pareyson.
Keywords: aesthetics, training, production, Luigi Pareyson, Gianni Vattimo

Palavras-chave: formatividade, produção, Luigi Pareyson, Gianni Vattimo 


\section{Introducción}

Formatividad y producción son los conceptos ejes sobre los cuales gira la renovación de la estética filosófica italiana a partir de la segunda mitad del siglo XX. El énfasis en el aspecto productivo de las obras de arte, por un lado, pretendía instalar la necesidad del recurso a la experiencia artística como conditio sine qua non para desarrollar una teoría estética y, por otro lado, preservar el carácter filosófico de la misma. La pregunta que sirve como telón de fondo para ese proceso de renovación estética no es, ciertamente, aquella que interroga por lo bello, la contemplación o las condiciones de posibilidad para emitir juicios de gusto, bien por el contrario, será aquella que, filosóficamente, a partir de las experiencias artísticas, se interroga por el proceso de formación y producción artística. Es en este punto que, desde nuestra perspectiva, la estética de la formatividad de Luigi Pareyson y de la producción de Gianni Vattimo se relacionan y potencian.

La formatividad estética, que según Pareyson es un hacer/producir/ inventar, pone el acento en un proceso productivo en el que no existe una forma artística antes de ser formada (forma formata). La forma artística será el fruto y la consecuencia del proceso de producción ( $\mathrm{Pa}$ reyson 2002a 75). Sin embargo, para Pareyson existe, una vez iniciado el proceso artístico, una forma formans presente activamente en ese proceso (Id. 76). En este sentido, no existiría un modelo desde el cual deducir las formas artísticas y las técnicas a ser empleadas por el artista en la producción de sus obras. Ese énfasis, también, muestra la necesidad de inventar las técnicas al mismo tiempo que se produce la obra artística. Algo similar acontece en la estética de la producción de Gianni Vattimo.

La producción de las formas artísticas, analizadas a partir de Aristóteles, conducen a Vattimo a afirmar la contingencia del arte, el carácter orgánico e histórico del proceso productivo y la necesidad de actualización de las formas artísticas a través del artista que actúa como causa eficiente de la misma. El acento en la producción artística implica, para Vattimo, la necesidad del recurso a las experiencias artísticas para pensar filosóficamente la estética. La relación entre estética de la formatividad y estética de la producción, entre Pareyson y Vattimo, aparece nítida.

La literatura filosófica sobre Gianni Vattimo tiende a colocar a la estética como el punto de partida y, en algunos casos, como llave maestra 
para una interpretación completa de la obra del filósofo italiano (Giorgio 2006). Otros, con buenas razones, tienden a mostrar la influencia de Pareyson en los textos estéticos de Vattimo (Amoroso 2008), al punto de afirmar que la tesis según la cual la estética tiene una vocación ontológica, defendida por Vattimo en su libro de 1967 Ontologia e poesia, sería una influencia pareysoniana. Concordando en lo substancial con Amoroso, podemos agregar que esa tesis aparece también en el libro de 1961 Il concetto di fare in Aristotele ${ }^{1}$.

En los apartados que siguen, presentamos las estéticas de la formatividad, de Luigi Pareyson, y de la producción, de Gianni Vattimo, a partir de sus textos originales. La finalidad de este artículo es afirmar la tesis según la cual la vocación ontológica de la estética en Gianni Vattimo, con una clara influencia de Pareyson, aparece también en la propuesta de una estética de la producción, a partir de Aristóteles, en su primera obra publicada en 1961 Il concetto di fare in Aristotele.

\section{Estética de la formatividad}

La estética filosófica de Luigi Pareyson es una teoría de la formatividad y de la producción. El libro de 1954, Estetica. Teoria della formatività, es el fruto maduro de una serie de investigaciones publicadas por Pareyson, en forma de artículos, en el arco de cuatro años. Para el investigador Francesco Paolo Ciglia, "la teoria della formatività nasce e si sviluppa rapidissimamente, fino ad assumere i suoi tratti fondamentali e la sua fisonomia definitiva, nel breve lasso di tempo compreso fa il 1950 ed il 1954"2 (123). La publicación sistemática de artículos en revistas italianas (Id. 123-124), y no solo, tenía el objetivo de formular una estética filosófica que fuera una alternativa diferente a lo que se producía en esa época en Italia.

El predominio hegemónico de la estética de Benedetto Croce en Italia, como sabemos, obliga a Luigi Pareyson a confrontarse cons-

\footnotetext{
1 Es importante, desde el punto de vista cronológico, tener en cuenta que ese libro es la tesis de Licenciatura en Filosofía que Gianni Vattimo defendió en la Università degli Studi di Torino, en 1959, bajo la guía de Luigi Pareyson.

2 "la teoría de la formatividad nace y se desarrolla rápidamente, hasta asumir sus trazos fundamentales y fisonomía definitiva, en el breve periodo de tiempo que va entre 1950 y 1954". Todas las traducciones al castellano de los textos originales italianos son de nuestra autoría.
} 
tantemente con esos planteamientos. Durante la segunta mitad de los años cuarenta del siglo pasado, como muestra la literatura italiana sobre ese asunto (Vattimo 2010 75; Givone 153-154; D'Angelo 179-188), la discusión con Benedetto Croce era una necesidad para todo aquel que escribiese sobre estética en Italia. Con todo, el objetivo de Pareyson no se reducía a una mera crítica de los principios fundantes de la estética crociana; por el contrario, su reflexión filosófica tenía un carácter programático de mayor alcance, que se materializa en la propuesta de una estética filosófica nueva denominada "teoría de la formatividad".

En las primeras líneas del prefacio a la edición de 1988, de Estetica. Teoria della formatività, Luigi Pareyson, contextualizando su estética, constata ese predominio de Benedetto Croce en la estética italiana. La constatación y crítica de ese predominio no es suficiente, porque se permanece dentro del esquema estético-filosófico crociano; se requiere, por consiguiente, una nueva propuesta estética. En las palabras de Pareyson:

L'estetica è il campo in cui il predominio di Croce è rimasto più a lungo incontrastato. Ancora nell'immediato dopoguerra l'estetica crociana era in Italia l'unica a cui si facesse riferimento [...] Invece di attardarsi in un'ennesima critica dell'estetica crociana, questo libro entrò subito in argomento, proponendo, al posto dei principi crociani dell'intuizione e dell'espressione, un'estetica della produzione e della formatività ${ }^{3}$ (2002a 7 en cursivas en el original).

La propuesta de Pareyson consiste en sustituir los principios estéticos crocianos de intuición y expresión por los de producción y formatividad. Este movimiento, realizado por Pareyson, posee un alcance mayor. Se trata, más propiamente, de sacar a la estética del ámbito científico y colocarla dentro de la filosofía. Es decir, la estética en Pareyson no es una disciplina científica, como pretendía Croce, sino más bien una filosofía del arte o, mejor aún, una estética filosófica.

3 "La estética es el campo en el que el predominio de Croce permaneció por más tiempo incontestado. Más aún, inmediatamente después de la Segunda Guerra Mundial la estética crociana era la única a la que se hacía referencia en Italia [...] En vez de demorarse en una enésima crítica a la estética crociana, este libro entró rápidamente en el argumento proponiendo, en el lugar de los principios crocianos de intuición y expresión, una estética de la producción y de la formatividad". 
Es importante y pertinente expresar aquí, que, para Benedetto Croce, "l'Estetica o è scienza dell'Espressione, o non trova posto in nessun luogo [...] una materia che sia propria dell'Estetica, fuori dell'Espressione, a nostro parere, non esiste" ${ }^{\prime 4}$ (81). El argumento crociano para reivindicar la cientificidad de la estética depende, necesariamente, de la aceptación del principio de expresividad de la materia como conditio sine qua non para la existencia de toda posible estética. La radicalidad del argumento se funda en el hecho de que, para Croce, la expresión presupone la impresión (Id. 1), esto es, la impresión sensible de una materia y, por lo mismo, "l'espressione o il fatto estetico esprime tutte le impressioni della realtà" ${ }^{5}$ (Id. 87). De este modo, la estética, en la perspectiva de Benedetto Croce, debe ser entendida como una ciencia capaz de expresar toda la realidad a partir de las impresiones de la materia. La realidad, para Benedetto Croce, puede ser expresada total y verdaderamente por la estética y, en este sentido, ésta última no puede ser sino ciencia. La estética como scientia capax, según el esquema de Croce, implica también que no es posible la interpretación y, menos aún, el error en el quehacer estético, elementos que, por el contrario, son fundamentales en la estética de Pareyson. En efecto, planteaba el filósofo valdostano:

la teoria del Croce rimane pur sempre estranea al riconoscimento della vera e propria «interpretazione», che ha un carattere insieme esecutivo e personale, e che consiste nell'esser non solo l'opera stessa qual essa vuol essere, ma anche, e al tempo stesso, l'espressione dell'interprete che così personalmente la possiede e la rende ${ }^{6}$ (Pareyson 1993 94).

La noción de "expresión", que difiere totalmente de aquella planteada por Croce, tiene para Pareyson una vinculación originaria con la interpretación. La obra de arte, en consecuencia, no es solo su materialidad, como afirma Croce, es, más propiamente, la expresión de

4 "la Estética o es ciencia de la Expresión, o no encuentra espacio en ningún lugar [...] una materia que sea propia de la Estética, fuera de la Expresión, en mi opinión, no existe". 5 "la expresión o el hecho estético expresa todas las impresiones de la realidad".

6 "la teoría de Croce permanece siempre extraña al reconocimiento de la propia y verdadera «interpretación», que tiene un carácter, al mismo tiempo, ejecutivo y personal, y que consiste en ser no solo la obra misma, como ella quiere ser, sino también, y al mismo tiempo, la expresión del intérprete que de este modo la posee y la ejecuta personalmente". 
la interpretación que el artista produce de sí y, al mismo tiempo, de la obra; la interpretación es ejecutora tanto de la obra como del ser de la persona que la realiza: a saber, el artista.

El movimiento ejecutado por Pareyson, con relación a la estética de Croce, nos permite concordar con lo expresado por Givone en Storia dell'estetica; es decir, que la estética de Pareyson puede ser definida como "post-crociana, più che anti-crociana"7 (Givone 153). La renuncia explícita de Pareyson, tal y como aparece en el prefacio de 1988 de Estetica. Teoria della formatività, a continuar criticando la estética de Croce, por un lado, confirma su no ser anti-crociana y, por otro lado, la realización de una estética centrada en la producción artística o formatividad la convierte, desde un punto de vista cronológico, en postcrociana.

La estética de la formatividad es aquella que realiza una síntesis entre la especulación o teoría filosófica y la experiencia artística concreta. De este modo,

(...) l'estetica non è una parte della filosofia, ma la filosofia intera concentrata sui problemi della bellezza e dell'arte, e in secondo luogo le questioni concrete dell'estetica per il fatto d'esser particolari non cessano affatto di esser filosofiche (...) L'estetica mostra chiaramente, appunto, che le due vie non si possono separare l'una dall'altra, giacché in filosofia l'esperienza è al tempo stesso oggetto di riflessione e verifica del pensiero, e il pensiero è al tempo stesso risultato e guida dell'interpretazione dell'esperienza ${ }^{8}$ (Pareyson 2002a 15-16).

El carácter filosófico de la estética no se pone en riesgo por el hecho de tener que confrontarse con la experiencia; por el contrario, es ella la que garantiza la subsistencia del pensamiento, por cuanto todo pensamiento filosófico es interpretación de una experiencia. Interpretación

7 "post-crociana, más que anti-crociana".

8 “(...) la estética no es una parte de la filosofía, sino la filosofía completa concentrada en los problemas de la belleza y del arte y, en segundo lugar, las cuestiones concretas de la estética, por el hecho de ser particulares, no dejan de ser filosóficas (...) La estética, justamente, muestra claramente que las dos vías no se pueden separar una de la otra, ya que en filosofía la experiencia es, al mismo tiempo, objeto de reflexión y verificación del pensamiento, y el pensamiento es, al mismo tiempo, resultado y guía de la interpretación de la experiencia". 
y experiencia son la conditio sine qua non para la subsistencia del pensamiento filosófico y estético. El carácter hermenéutico de toda filosofía, para Pareyson (Uribe Miranda 2014a), impregna también toda estética (Uribe Miranda 2014b), porque sin interpretación nada puede ser expresado y comprendido. Que la estética sea filosófica no significa renunciar a la experiencia artística; por el contrario, es la interpretación de la experiencia artística la que torna filosófica a la estética. En palabras de Pareyson:

Bisogna dunque riconoscere che l'estetica è filosofia, e solo a patto d'esser filosofia giustifica la propria autonomia; ma bisogna insieme riconoscere che ciò non vuol dire affatto ch'essa debba perdersi nelle nubi della sterile astrattezza e disertare la frequentazione dell'esperienza. L'estetica, come del resto tutta la filosofia, ha un carattere insieme concreto e speculativo (...) L'estetica è costituita da questo duplice richiamo al carattere speculativo della riflessione filosofica e al suo vitale contatto con l'esperienza: non è estetica quella riflessione che, non alimentata dall'esperienza dell'arte e del bello, si riduce a mero gioco verbale, né quell'esperienza d'arte o di bellezza che, non elaborata su un piano speculativo, rimane semplice descrizione $^{9}$ (2002a 17 ).

El reconocimiento del carácter filosófico de la estética no significa, para Pareyson, perderse en reflexiones estériles y abstractas, bien por el contrario, significa que la estética es filosofía porque ella necesita tanto de la especulación como de la experiencia vital. Los dos elementos enunciados no se separan ni jerarquizan en la estética, a riesgo de transformar la estética en estéril especulación o mera descripción de obras de arte. El desafío, por tanto, consiste en mantener unidas la especulación y la experiencia para que la estética pueda desarrollarse como tal, esto es, como filosofía.

$9 \quad$ "Es necesario, entonces, reconocer que la estética es filosofía y la solo condición de ser filosofía justifica su autonomía; pero es necesario reconocer, también, que eso no quiere decir que de hecho ella deba perderse en las nubes de la abstracción estéril y abandonar la frecuentación de la experiencia. La estética, como por lo demás toda la filosofía, tiene un carácter concreto y especulativo al mismo tiempo (...) La estética está constituida de este doble reclamo al carácter especulativo de la reflexión filosófica y su contacto vital con la experiencia: no es estética aquella reflexión que, no alimentada de la experiencia del arte y de lo bello, se reduce a mero juego verbal, ni tampoco aquella experiencia del arte o de la belleza que, no elaborada sobre un plano especulativo, permanece simple descripción". 
La filosofía tiene, para Pareyson, un carácter personal que, sin embargo, no puede ser confundido o mal interpretado como un carácter subjetivo. Los conceptos de "persona" y "sujeto" no pueden ser homologados en la filosofía pareysoniana porque, justamente, el personalismo ontológico, que afirma la preeminencia ontológica de la persona, se opone al racionalismo moderno, que afirma la preeminencia del sujeto. La crítica al sujeto moderno y a la modernidad como filosofía, en detrimento de la persona, permite a Pareyson desarrollar una filosofía de la persona que encuentra en la interpretación y la experiencia vital sus fundamentos. En Il compito della filosofia oggi, texto originalmente escrito en 1947, Pareyson afirma que "la filosofia è approfondimento, interpretazione, esplicazione di una singola prospettiva personale"10 (2002b 146), y agrega después que "la filosofia è veramente speculativa e scopre un valore assoluto di verità attraverso l'interpretazione personale che il filosofo dà di sé e insieme della realtà" ${ }^{11}$ (Id. 147). El argumento de que la filosofía es interpretación personal de la realidad, desde nuestra perspectiva, solo refuerza el carácter filosófico de la estética como necesitada tanto de la especulación como de la experiencia.

Que la estética necesite y se nutra de la experiencia artística no hace más que poner de manifiesto el carácter operativo de la persona. La persona es un ser activo. Es decir, la creación artística es fruto de un obrar, de una actividad humana que, en términos pareysonianos, se expresa a través del verbo italiano fare. Fare se traduce literalmente en castellano como hacer, pero en Pareyson adquiere, desde el punto de vista estético, el sentido de producción e invención simultánea. Es justamente desde esta constatación experiencial del arte, como producción e invención, que Pareyson toma el adjetivo para su estética: la formatividad. La formatividad se define como "un tal «fare» che, mentre fa, inventa il «modo di fare»: produzione ch'è, al tempo stesso e indivisibilmente, invenzione"12 (Pareyson 2002a 18). Enunciada la formatividad, queda claro, para Pareyson, que el proceso artístico

10 "la filosofía es profundización, interpretación, explicación de una individual perspectiva personal".

11 "la filosofía es verdaderamente especulativa y descubre un valor absoluto de verdad a través de la interpretación personal que el filósofo da de sí mismo y, en ella, de la realidad".

12 "un tal «hacer» que, mientras hace, inventa el «modo de hacer»: producción que es, al mismo tiempo e indivisiblemente, invención". 
es, al mismo tiempo, como un proceso formativo. Crear es formar y, según esto, la formación de la obra de arte "è un puro tentare" ${ }^{13}$ (Id. 69) que no posee de antemano la forma a crear y donde la obra artística se forma en la "simultaneità d'invenzione ed esecuzione" ${ }^{14}$ (Id. 72). En otras palabras, que no existe una forma ejemplar que sirva de modelo para producir la obra de arte como $\mu$ í $\eta \sigma \iota \varsigma$ o imitatio. Por el contrario, es en la ejecución sin norma previa y fruto de un hacer/producir que inventa su propio modo de hacer/producir, lo que, empero, no implica una ausencia total de una guía ( $I d$. 70), donde se hace presente la obra de arte como creación. La obra artística es una forma formata a partir de un hacer/producir/inventar, en el que, una vez iniciado el proceso de producción, está presente activamente una forma formans (Id. 76), esto es, de una formatividad. En consecuencia, la estética no puede ser sino el resultado de una experiencia artística que, a su vez, es la materia prima de la reflexión filosófica que es, por un lado, interpretación y, por otro, especulación.

La estética para Pareyson, según lo hasta aquí expuesto, posee un carácter especulativo en cuanto es filosófica y no histórica en el sentido de las poéticas. A este respecto afirma Pareyson:

affermare il carattere speculativo dell'estetica non significa disconoscere o negare la storicità. Ma la sua storicità non è quella delle poetiche, che si susseguono, si alternano e si combattono nella storia dei fatti artistici (...) bensì è la storicità della filosofia, nella quale la molteplicità delle dottrine non compromette, ma ribadisce la fondamentale unità del pensiero filosofico ${ }^{15}$ (2002a 317).

En otras palabras, que la estética es especulativa porque asume su carácter histórico, no al modo de las poéticas, sino al modo de la filosofía, esto es, como reflexión de las experiencias artísticas, manifestando de esta manera, para Pareyson, la unidad y multiplicidad de la filosofía, como había preconizado en la introducción a su trabajo sobre Fichte

\footnotetext{
13 "es un puro intentar".

14 "simultaneidad de invención y ejecución".

15 "afirmar el carácter especulativo de la estética no significa desconocer o negar la historicidad. Sin embargo, su historicidad no es aquella de las poéticas, que se subsiguen, se alternan y se combaten en la historia de los hechos artísticos (...) pero que es la historicidad de la filosofía, cuya multiplicidad de las doctrinas no compromete, sino que más bien confirma la fundamental unidad del pensamiento filosófico".
} 
en 1959, tal y como hemos mostrado en otro escrito (Uribe Miranda 2014a 237-243).

Una interpretación racionalista de la filosofía, in extremis, podría llevarnos al equívoco de pensar que la estética, por ser filosófica, no guarda relación con la vida y sí con la pura razón, proponiendo una disociación entre arte y vida. A este respecto se puede agregar que el existencialismo, desde el cual Pareyson formula su personalismo ontológico, en conjunto, es un antecedente ineludible de su teoría estética. Estos antecedentes permiten a Pareyson afirmar, indubitablemente, que no existe una disociación entre arte y vida o, si se quiere, entre estética filosófica y vida. Para el filósofo valdostano, "tutta la vita è già «arte» e che l'arte proprio in quanto tale è sempre "più» che arte"16 (2002a 275). En ese più che arte (más que arte) se expresa el carácter formativo de la existencia humana en cuanto acción creadora, formativa, que al mismo tiempo forma también la existencia de la persona. El hacer/producir/inventar del artista en su obra es, al mismo tiempo, expresión formativa del ser personal del artista. Por tanto, la filosofía, como el arte, también es una acción formativa en la que está involucrada e implicada la totalidad de la existencia de la persona (Id. 275-276) y que, en consecuencia, la afirmación de una estética filosófica no implica la afirmación de una estética racionalista desvinculada de la vida.

El concepto de "formatividad" en la estética de Luigi Pareyson pone de manifiesto el carácter filosófico de la estética asociando arte y vida, pero también nos permite afirmar que ese concepto pone el énfasis en la producción de las obras artísticas. Formatividad y producción se imbrican a tal punto en la estética de Luigi Pareyson, que ya no es posible pensar la estética filosófica sin referencia a la experiencia artística concreta y que la sola descripción de obras artísticas, sin referencia a la reflexión filosófica, tampoco puede ser denominada propiamente "estética".

\section{Estética de la producción}

Las temáticas estéticas son una constante en las investigaciones filosóficas de Gianni Vattimo en sus primeros años de formación fi-

16 “toda la vida es ya «arte» y que el arte, justamente, en cuanto tal, es siempre «más» que arte". 
losófica. Como es sabido, al menos en la literatura italiana, la etapa de estudios del joven Gianni Vattimo en la Universidad de Turín, de mediados de los años cincuenta del siglo pasado, está fuertemente marcada por la figura de su maestro Luigi Pareyson. La influencia de Pareyson conduce a Vattimo a pensar la cuestión estética en términos de producción y no tanto de expresión, como solía ser común en la Italia de esa época, al punto de decidirse a investigar la importancia del hacer como producción a partir de Aristóteles. Esa influencia se materializa en la publicación del primer libro de Vattimo, en 1961, cuyo título es Il concetto di fare in Aristotele. La publicación de ese libro, así como de otros artículos sobre estética publicados en 1960 (Vattimo 2008a; 2008b; 2008c), ha llevado a algunos de sus intérpretes a pensar que la estética es la llave maestra para comprender la obra vattimiana (Giorgio 25-31).

La influencia que Pareyson ejerce sobre Vattimo es fundamentalmente filosófica. Es decir, que asume de modo activo la influencia del maestro para afirmar la pertinencia de la estética de la formatividad y, con ello, abrir nuevas posibilidades de lecturas sobre la obra del Estagirita, por lo menos en Il concetto di fare in Aristotele.

En términos filosóficos, Vattimo pretendía salir de las interpretaciones románticas de Aristóteles, en particular de los conceptos de $\mu i ́ \mu \eta \sigma ı \varsigma$ y кá $\theta \alpha \rho \sigma ı \varsigma$ a partir de Butcher e Bernays, que habían marcado a fuego las interpretaciones sobre la estética aristotélica hasta la segunda mitad de la década del cincuenta del siglo pasado (Vattimo 2008a 202-203). El intento vattimiano consistirá en confirmar las reflexiones de Pareyson presentes en la teoría de la formatividad, centrándose sobre la cuestión de la contingencia de la forma en Aristóteles y, por lo mismo, pensar la producción artística en términos de organicidad de la forma; es decir, abrirse a la tarea de ordenar lo contingente en términos de mundo histórico (Cedrini, Martinengo y Zabala 14). La contingencia de la forma artística, a partir de Aristóteles, conduce a Vattimo a pensar una estética de la producción en diálogo fecundo con Pareyson.

Gianni Vattimo comienza su libro Il concetto di fare in Aristotele con una declaración metodológica, con el fin de definir el lugar del arte entre las actividades humanas según Aristóteles. Con este propósito, Vattimo afirma que: 
La definizione della $\tau \varepsilon ́ \chi v \eta$ che Aristotele dà nel libro VI dell'Etica Nicomachea costituisce il punto di partenza della nostra ricerca, sia perché serve a inquadrare l'arte fra le altre attività dell'uomo, sia perché, per la sua precisione, delimita il campo in cui ci dovremo muovere, sia infine perché, analizzata nei particolari, contiene una esauriente indicazione dei problemi che dovremo affrontare ${ }^{17}$ (2007 21).

La declaración metodológica permite a Vattimo realizar un inte-

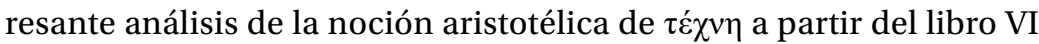
de la Ética Nicomaquea. Como es sabido, en este libro se plantea la división del alma en una parte racional ( $\lambda$ ó $\gamma \circ v$ Ě $\chi o v)$ y otra irracional

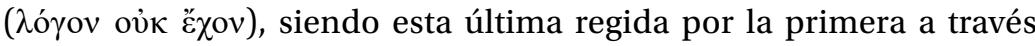
de las virtudes éticas y la primera por las virtudes dianoéticas. Además, la parte racional se subdivide en dos partes: por un lado, la parte $\dot{\varepsilon} \pi \_\tau \eta \mu$ ovıкóv, que dice relación con lo necesario, y, por otro, la parte

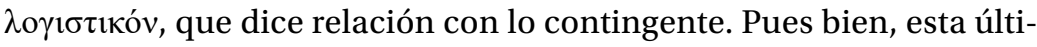

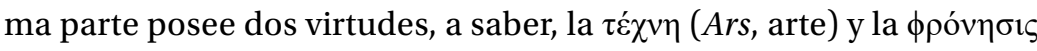
(Prudencia) o, en palabras de Vattimo, que "Le virtù del contingente,

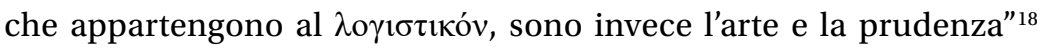
(Ibíd.). Con todo, existiría una diferencia entre el arte y la prudencia, aunque ambas sean medios o formas del alma para expresar o estar en relación con la verdad, que es proporcional a la diferencia entre hacer y obrar. Como explica Vattimo:

La differenza fra arte e prudenza è la stessa che intercorre tra il fare e l'agire: l'arte riguarda infatti la produzione di oggetti diversi da chi fa, mentre l'agire rappresenta una attività intransitiva, per così dire, che rimane in chi la fa; la perfezione dell'agire è la perfezione stessa dell'agente, mentre il produrre ha il suo atto in un certo senso fuori dal soggetto ${ }^{19}$ (2007 22).

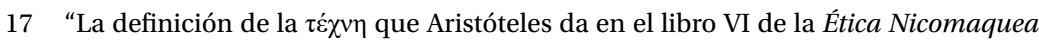
constituye el punto de partida de nuestra investigación, sea porque sirve para encuadrar el arte entre las otras actividades del hombre, sea porque, por su precisión, delimita el campo en que nos deberemos mover, sea porque finalmente, analizada en sus particulares, contiene una exhaustiva indicación de los problemas que deberemos enfrentar".

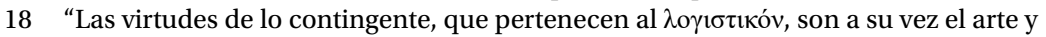
la prudencia".

19 "La diferencia entre arte y prudencia es la misma que hay entre el hacer y el obrar: el arte, de hecho, refiere a la producción de objetos diferentes de quien los haga, mientras que el obrar representa una actividad intransitiva, por así decir, que permanece en 
El obrar implica una perfección del agente al realizar una acción, porque su fin está in se y se distingue del hacer, porque su acción está dirigida a la producción de objetos externos o, en otros términos, que

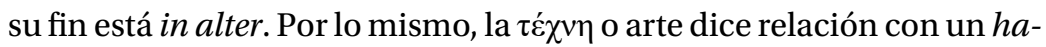

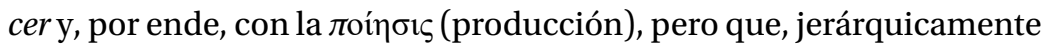
desde el punto de vista de la perfección, están regidas por la prudencia. En otras palabras, el arte, por lo menos desde la Ética Nicomaquea, es un hacer como producción que posee un estatus inferior e instrumental respecto del obrar. El obrar virtuoso, al contrario del hacer que se agota en la producción de objetos, está ordenado al fin último de la

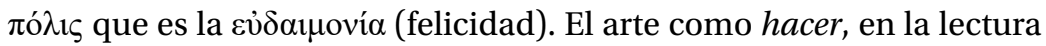
de Vattimo, interpretando a Aristóteles, "non ha un valore autonomo" ${ }^{20}$ (Id. 26), porque su valor depende del uso. El producto artístico adquiere en Aristóteles el carácter de utensilio cuyo valor está en su uso o, mejor, en su utilidad, lo que implica una acción manual que, desde la perspectiva aristotélica, significa poseer un rango menor.

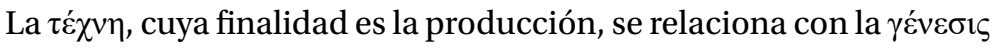
(origen). Esto es evidente en la medida que el hacer como producción se refiere a la generación de un objeto en vistas a su uso. El análisis vattimiano, ahora a partir del II libro de la Física de Aristóteles, se dirige

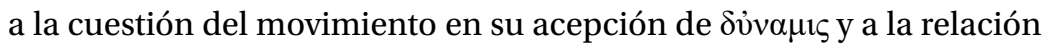
de este con la $\mu$ í $\eta$ бıৎ (imitación). En otros términos, se postula que existiría una relación entre $\tau \dot{\chi} \chi \vee \eta$ y $\phi u ̉ \sigma ı$, , porque el arte debe ser capaz de originar, como producción, una obra que sea imitación de la naturaleza: un hacer que haga (produzca) en un movimiento, como hace la naturaleza (Vattimo 2007 40). En este sentido, el arte es el principio del movimiento de generación según la naturaleza. Sin embargo, aquí se abre para Vattimo un problema respecto de la comprensión que podemos tener de la imitación. Es decir, ¿cómo el artista es capaz de dar forma ( $\varepsilon \tilde{i} \delta o \varsigma)$ a una obra? ¿Cuál sería la naturaleza de la imitación respecto de su original? Estas interrogantes obligan a Vattimo a tener que analizar el concepto de "imitación" en el sentido aristotélico presente en la Poética. Es aquí donde se redescubre para Vattimo la relación en-

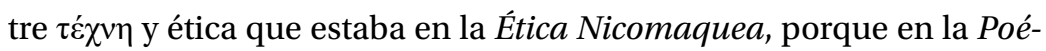

quien la hace; la perfección del obrar es la perfección misma del agente, mientras que el producir tiene su acto, en cierto sentido, fuera del sujeto".

20 "no tiene un valor autónomo". 
tica la imitación por excelencia es la tragedia, justamente porque es imitación del mundo moral humano sobre la base de la construcción del mito. De este modo, el poeta es un mitómano, no un mentiroso, sino un generador de mitos según las reglas de la imitación, un artífice de mitos, un artífice que se mueve entre el placer y la catarsis. Sin embargo, para Vattimo, estas tragedias y mitos tienen una naturaleza dinámica en Aristóteles (Id. 72), esto es, que no permanecen estáticas e invariantes, sino que, según las reglas morales (porque la tragedia compete a los hombres buenos mientras que la comedia compete a los malos ${ }^{21}$ ), van dando forma a la tragedia.

Teniendo como telón de fondo los conceptos de "tragedia" y de "producción estética", Gianni Vattimo se concentra sobre la cuestión de la generación de la forma del producto artificial. Con todo, aparece aquí un problema que el filósofo italiano plantea en los siguientes términos:

Se si rinuncia a spiegare tutta l'arte secondo il concetto di imitazione-riproduzione come a noi sembra si debba fare - poiché è indiscutibile che per Aristotele ci sono anche arti non imitative, che non riproducono cioè oggetti e processi visti in natura - ci si trova di fronte al grave problema della nascita della forma artistica ${ }^{22}$ (Vattimo 2007 75).

La existencia de obras artísticas, para Aristóteles, que no son imitación de la naturaleza, lleva a Vattimo a preguntar por el surgimiento de la forma artística. El problema se torna más complejo, según Vattimo, cuando debemos aceptar que en Aristóteles no existe el concepto de "creación". Este problema, sin embargo, al contrario del concepto, común en la estética moderna, de "creación artística", abre el camino para pensar una filosofía de la producción "che appunto non è mai filosofia della creazione"23 (Vattimo 2007 76). Como sabemos, para Aristóteles, en la Metafísica, al contrario de Platón, todo lo que se genera está com-

21 Argumento a partir del cual Umberto Eco escribirá el ya celebre texto Il nome della rosa, en 1980.

22 "Si se renuncia a explicar todo el arte según el concepto de imitación-reproducción como nos parece que se deba hacer - porque es indiscutible que para Aristóteles existen artes no imitativas, que no reproducen objetos y procesos vistos en la naturaleza- nos encontramos de frente al grave problema del nacimiento de la forma artística".

23 "que justamente no es nunca filosofía de la creación". 
puesto de materia y forma, de modo tal que no preexiste una forma que informe a la materia (Id. 77). La forma, que no está separada de la materia, posee valor de causa que la hace ser acto de una sustancia particular (Id. 84). En otras palabras, la forma artística en Aristóteles no es un arquetipo estable, sino que llega a ser en un proceso productivo de materia y forma, y acto y potencia. Es aquí donde Vattimo manifiesta su adhesión con la interpretación de Aristóteles realizada por su maestro Luigi Pareyson. Es decir, la forma de la obra de arte solo se realiza en el hacer y es en este que tiene sentido (Pareyson 2002a 57). La forma solo toma acto en el hacer (Vattimo 2007 84). El concepto de " $\tau \varepsilon \dot{\varepsilon} \chi v \eta$ ", en la medida en que es entendido por Aristóteles, por un lado, en la Ética Nicomaquea como una virtud, es decir, como una fuerza o disposición para actuar y producir, y, por otro, en la Metafísica a partir de su distinción de la ciencia, puede ser comprendido como una

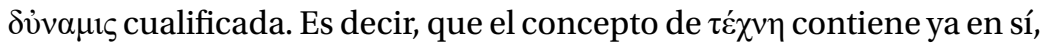
en cuanto $\delta \dot{v} v \alpha \mu \varsigma$ cualificada, una actualidad. La $\delta \dot{v} v \alpha \mu \varsigma$ cualificada es un acto (Id. 93) y nos permite afirmar que la $\tau \dot{\varepsilon} \chi v \eta$, esa $\delta v ̉ v \alpha \mu 1 \zeta$ cualificada, puede producir las formas artísticas porque, en cuanto acto, ella

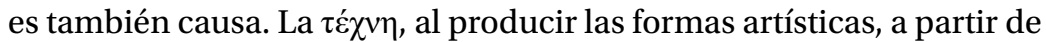
Aristóteles, niega el concepto de "creación".

El concepto de " $\tau \dot{\varepsilon} \chi \vee \eta$ " permite a Vattimo comprender el proceso productivo de las obras artísticas. Este proceso es inseparable de la noción de "movimiento", toda vez que producir algo es ponerlo en movimiento; es decir, que algo pasa de la potencia al acto. Ahora bien, estos movimientos están en perspectiva de la causa formal, de la finalidad y contingencia, pero, sobre todo, de un movimiento orgánico que incluye tanto el producto como el proceso mismo de producción. Para Vattimo, reforzando lo dicho, "questa organicità non è però propria soltanto dell'oggetto prodotto, bensì appartiene anche e prima di tutto il processo"24 (2007 134-135). Existiría un proceso orgánico que, por consecuencia, otorga esa organicidad al producto u obra artística. No existiría obra artística que no sea un organismo (Id. 147). Sin embargo, la noción de "organismo", que primeramente hace referencia al movimiento in se, es decir a los cuerpos con alma, vuelve proble-

24 "esta organicidad no es, con todo, propia solo del objeto producido, sino que pertenece también y antes de todo al proceso". 
mática la referencia a la obra de arte. Con todo, este problema se resuelve con la introducción del concepto de "substancia", toda vez que Aristóteles refiere esta noción tanto a los organismos naturales como a los artificiales en cuanto ambos son substancia. Además, en el caso de las producciones artificiales o artísticas el movimiento viene dado por su ser instrumentos de producción o de uso y, en este sentido, el movimiento viene dado por el productor o por el que utiliza el instrumento; esto es, que "il concetto di strumento rimane quello essenziale e fondamentale" ${ }^{25}$ (Id. 151) porque "l'organismo come strumento ha un movimento che è vita" ${ }^{26}$ (Id. 152). La producción artística es fruto de un organismo vivo en el que los instrumentos cumplen una función orgánica en la totalidad del proceso productivo. Por tanto, los caracteres formales de unidad, totalidad y perfección que se atribuyen a las obras de arte, a partir de Aristóteles, se conectan con la vida porque se dicen del organismo ya que éste, también, es un instrumento del alma (Id. 164).

A tenor de lo anterior, Vattimo planteará que:

Insomma in Aristotele il prodotto, anche dell'arte bella, è strumento non di comunicazione, bensì d'uso: se comunicazione c'è, essa o è subordinata o avviene proprio lungo quelle linee di invarianza delle caratteristiche dell'uomo e, dentro quest'ambito mai definito una volta per tutte, secondo quelle modifiche, quei ritorni, quei mutamenti che sono propri della storia della cultura ${ }^{27}$ (2007 174).

La producción artística es un instrumento de uso, dada su vinculación con el hombre y, en él, con la historia de la cultura. Es justamente por este aspecto que las obras del arte y de la técnica adquieren un carácter activo, carácter pensado por Vattimo en términos de una ineludible vinculación con la historia. Como bien afirma Vattimo, "il mondo della $\tau \varepsilon ́ \chi v \eta$, visto così, è lo stesso mondo della storia, e l'arte è

\footnotetext{
25 "el concepto de instrumento permanece como esencial y fundamental".

26 "el organismo como instrumento tiene un movimiento que es vida".

27 "En Aristóteles, en definitiva, el producto, también el de las bellas artes, es instrumento no de comunicación sino de uso: si la comunicación existe, ella o está subordinada o adviene justamente a través de esas invariables líneas características del hombre y, dentro de este ámbito nunca definido de una vez y para siempre, según esas modificaciones, esas vueltas, esas mutaciones que son propias de la historia de la cultura".
} 


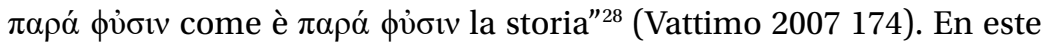
sentido, la estética para Vattimo no tiene una vinculación puramente contemplativa o de comunicación, ella adquiere también una dimensión activa e histórica porque se dice de lo contingente. No se podría, por tanto, pensar la estética como una pura ciencia de la expresión o, si se quiere, de la contemplación, porque equivaldría a ponerla en el ámbito de lo necesario olvidando su carácter de $\tau \dot{\chi} \chi v \eta$. La estética, ya en el planteamiento del joven Vattimo, viene pensada como una filosofía del arte de la producción, y se vincula necesariamente con la historia,

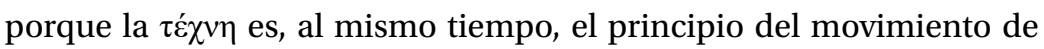
lo singular y del devenir del todo. Por tanto, la estética, a partir de la lectura de Aristóteles, viene pensada por Vattimo como una estética de la producción histórica, activa y contingente de las obras artísticas.

\section{Conclusión}

"Formatividad" y "producción" han sido los conceptos a partir de los cuales hemos presentado las estéticas filosóficas de Luigi Pareyson y Gianni Vattimo. Ambas propuestas parten del mismo diagnóstico; a saber, que la estética filosófica no puede continuar teniendo como objetivo la expresividad de las obras de arte, la contemplación de lo bello y, además, tener la pretensión de ser ciencia. Las estéticas de Pareyson y Vattimo, saliendo del esquema propuesto por Benedetto Croce en Italia e incorporando otros referentes teóricos, se presentan como estéticas filosóficas de la producción artística.

La centralidad que adquiere en Vattimo el concepto estético de "producción", sea en su versión aristotélica como en su versión heideggeriana posterior, muestran con suficiente claridad la influencia ejercida por Luigi Pareyson en sus primeros años de formación. El reconocimiento recurrente de Vattimo a la figura señera de Pareyson, lo lleva a afirmar en muchas ocasiones que éste es "certamente uno dei pensatori che più hanno segnato la filosofia italiana di questi ultimi decenni"29 (Vattimo 1991 49).

28 "el mundo de la $\tau \varepsilon ́ \chi v \eta$, visto así, es el mismo mundo de la historia, y el arte es $\pi \alpha \rho \alpha ́$

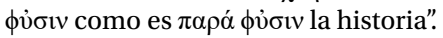

29 "ciertamente uno de los pensadores que marcaron más la filosofía italiana de estos últimos decenios". 
El concepto pareysoniano de "formatividad" es interpretado, a partir de Aristóteles, como producción por Vattimo, mostrando de este modo la influencia y continuidad filosófica que existe entre ambos filósofos. Este, nos parece, es el espíritu de las afirmaciones de Leonardo Amoroso respecto del primer libro de estética de Vattimo. Dice Amoroso:

il fatto che fin dal titolo sia evidente un'influenza dell'estetica di Pareyson, alla quale, per certi versi, Vattimo rilegge Aristotele, è senz'altro spiegabile come un omaggio al maestro da parte del giovanissimo allievo, ma ha anche un suo interesse filosofico che è opportuno sottolineare: un'estetica come quella di Pareyson può ben svolgere questo ruolo di riferimento utile per comprendere un'«estetica» precedente all'estetica come filosofia dell'arte bella, perché essa stessa , l'estetica di Pareyson, è qualcosa di diverso e di più rispetto a un'estetica intesa come filosofia dell'arte bella ed è invece caratterizzata, anche grazie ai suoi legami con l'esistenzialismo e con l'ermeneutica, da una decisiva vocazione ontologica ${ }^{30}$ (2008 15).

La indicación de Amoroso no es menor. Ella nos permite enunciar que, por un lado, la estética de la producción de Vattimo tiene una vocación ontológica, como aparece nítidamente en su libro Ontologia e poesía, y, por otro lado, que la estética de la formatividad de Pareyson es una referencia para comprender e interpretar las estéticas precedentes entendidas como filosofías de las bellas artes, porque, justamente, tiene una vocación ontológica. Lo planteado por Amoroso, según el cual "appunto la rivendicazione di una vocazione ontologica dell'estetica è l'istanza di fondo fatta valere da Vattimo in Poesia e ontologia, richiamandosi a Pareyson" ${ }^{31}$ (2008 15), nos permite aceptar y ampliar esa

30 "el hecho de que, desde el título, exista una evidente influencia de la estética de Pareyson, a la cual, en ciertos momentos, Vattimo relee Aristóteles, es sin dudas explicable como un homenaje al maestro por parte del joven alumno, pero también tiene su interés filosófico que es oportuno subrayar: una estética como la de Pareyson puede desarrollar bien ese rol de referencia útil para comprender una «estética» precedente a la estética como filosofía de las bellas artes, porque ella también, la estética de Pareyson, es algo diferente, y más con respecto a una estética entendida como filosofía de las bellas artes, y está a su vez caracterizada, también gracias a sus lazos con el existencialismo y con la hermenéutica, por una decidida vocación ontológica".

31 "justamente la reivindicación de una vocación ontológica de la estética es la instancia de fondo que Vattimo sostiene in Poesia e ontologia, remitiéndose a Pareyson". 
interpretación. Nuestra tesis, anunciada en la introducción de este artículo, de que la vocación ontológica de la estética en Gianni Vattimo, con una clara influencia de Pareyson, aparece también en la propuesta de una estética de la producción, a partir de Aristóteles, en su primera obra publicada en 1961, Il concetto di fare in Aristotele, puede ser confirmada a partir de lo siguiente. En primer lugar, porque el concepto de "producción" es analizado por Vattimo a partir de Aristóteles. El concepto a partir del cual Vattimo afirma su propuesta estética es el

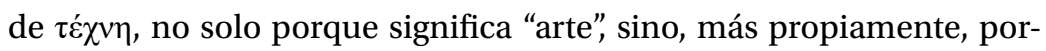
que este remite a la producción de las formas artísticas en el ámbito de lo contingente. Con base en ese análisis, Vattimo llega a la conclusión de que las formas artísticas no son arquetipos o esencias separadas; al contrario, son radicalmente históricas y culturales porque remiten al hombre en cuanto artista que, a su vez, es la causa eficiente que permite el paso de la potencia al acto de dichas formas artísticas. El carácter contingente del arte y de las producciones artísticas solo puede ser afirmado desde un punto de vista ontológico. La pregunta por el ser de las formas artísticas en su proceso de producción en Il concetto di fare in Aristotele de Vattimo, manifiesta la vocación ontológica de la estética. Vocación ontológica que, a partir de la estética de la formatividad de Pareyson, Vattimo asume y desarrolla con propiedad.

En segundo lugar, porque es evidente que el concepto de "formatividad" en Pareyson remite a una estética del proceso de formación y producción artística que supone una ontología. Como mostramos en el primer apartado de este texto, la estética como teoría de la formatividad de Pareyson es consecuencia del personalismo ontológico y de una ontología de lo inagotable. El ser para Pareyson es inagotable, y es esa justamente la razón por la cual las obras de arte pueden ser interpretadas sin por ello agotarse. Para Pareyson, el carácter hermenéutico de las obras de arte las vuelve, al mismo tiempo, ontológicas. La vocación ontológica de la estética de la formatividad toma fuerza en el concepto de "forma", una forma que es fruto de un hacer/producir/inventar (forma formata) al mismo tiempo, y que, para Pareyson, implica la existencia de la forma formans que ya actúa desde el inicio del proceso de producción. Desde aquí, en nuestra lectura, Gianni Vattimo, por un lado, confirma la pertinencia de la estética pareysoniana utilizando la idea de "forma" y "producción", yendo a Aristóteles para afirmar su es- 
tética con vocación ontológica a partir del carácter contingente e histórico de las formas artísticas y, por otro lado, abre nuevas posibilidades para futuras lecturas filosóficas de la obra estética de Aristóteles, tal y como mostramos en el segundo apartado de este artículo.

En tercer lugar, porque Gianni Vattimo y Luigi Pareyson defienden el carácter filosófico de la estética. El hecho de que la estética sea filosófica no significa descartar las experiencias artísticas concretas; al contrario, para ambos el recurso a las experiencias artísticas concretas es la materia prima para toda reflexión estético-filosófica. La estética filosófica supone y depende de las experiencias artísticas concretas; sin embargo, esto no significa que la sola experiencia artística concreta sea suficiente para decretar el carácter estético de esa reflexión. La afirmación del carácter filosófico de la estética manifiesta la vocación ontológica de la misma y que, como hemos afirmado, ya está presente en la estética de la producción de Vattimo a partir de Aristóteles. La pregunta realizada por Vattimo acerca del origen de las formas artísticas en Aristóteles, le permite no solo afirmar el carácter histórico de las formas artísticas, sino que, más precisamente, lo habilita para afirmar que ontológicamente todas las formas artísticas tienen un origen natural y que, por tanto, la estética de la producción se ejecuta secundum natura, revelando así su vocación ontológica.

"Formatividad" y "producción" son los conceptos que, a partir de Pareyson y Vattimo, nos permiten afirmar el carácter filosófico de la estética y su connatural vocación ontológica, como hemos mostrado a lo largo de este escrito.

\section{Bibliografía}

Amoroso, Leonardo. "Gianni Vattimo e il primato "debole" dell'estetica", Pensare l'attualità, cambiare il mondo. Confronto con Gianni Vattimo. Org. Gaetano Chiurazzi. Milán: Ed. Mondadori, 2008. 11-22.

Cedrini, Mario, Martinengo, Alberto y Zabala, Santiago. "Presentazione”, Opere Complete. Vattimo, Gianni, Vol. I, Tomo 1. Roma: Ed. Meltemi, 2007. 9-16.

Ciglia, Francesco Paolo. Ermeneutica e libertà. L'itinerario filosofico di Luigi Pareyson. Roma: Bulzoni, 1995. 
Croce, Benedetto. Tesi fondamentali di un'estetica come scienza dell'espressione e linguistica generale. Nápoles: Ed. Bibliopolis, 2002.

D’Angelo, Paolo. L'estetica italiana del novecento. Roma-Bari: Ed. Laterza, 2007.

Giorgio, Giovanni. Il pensiero di Gianni Vattimo. Milán: Ed. Franco Angeli, 2006.

Givone, Sergio. Storia dell'estetica. Roma-Bari: Ed. Laterza, 2008.

Pareyson, Luigi. Estetica. Teoria della formatività. Milán: Ed. Bompiani, 2002a.

Pareyson, Luigi. Prospettive di filosofia contemporanea. Milán: Ed. Mursia, 1993.

Pareyson, Luigi. "Il compito della filosofia oggi". Esistenza e persona. Génova: Ed. Il melangolo, 2002b. 135-150.

Uribe Miranda, Luis. "Interpretatio Prima. La primera formulación de una filosofía de la interpretación en Luigi Pareyson", Hermenéutica Intercultural: Revista de Filosofía 23 (2014a): 229-247.

Uribe Miranda, Luis. "Estética e Interpretación. La segunda y tercera formulación de una filosofía de la interpretación en Luigi Pareyson", Hybris. Revista de Filosofía 5/2 (2014b): 75-93.

Vattimo, Gianni. Introduzione all'estetica. Org. Leonardo Amoroso. Pisa: Ed. ETS, 2010.

Vattimo, Gianni. "Imitazione e catarsi in alcuni recenti studi aristotelici”, Opere Complete. Vol. I, Tomo 2. Roma: Ed. Meltemi, 2008a. 201-210.

Vattimo, Gianni. "Opera d'arte e organismo in Aristotele", Opere Complete. Vol. I, Tomo 2. Roma: Ed. Meltemi, 2008b. 211-233.

Vattimo, Gianni. "Per una storia dell'estetica", Opere Complete. Vol. I, Tomo 2. Roma: Ed. Meltemi, 2008c. 235-241.

Vattimo, Gianni. Il concetto di fare in Aristotele, Opere Complete. Vol. I, Tomo 1. Roma: Ed. Meltemi, 2007. 19-180.

Vattimo, Gianni. Etica dell'interpretazione. Turín: Ed. Rosenberg \& Sellier,1991. 